\title{
Perfil de motociclistas que utilizam equipamentos de proteção individual na Região Metropolitana de Recife, Brasil
}

Profile of motorcyclists using personal protective equipment in the Metropolitan Region of Recife, Brazil

Perfil de motociclistas que utilizan equipo de protección personal en la Región Metropolitana de Recife, Brasil

Rennan Luiz Oliveira dos Santos ${ }^{1}$ ORCID: https://orcid.org/0000-0001-9093-3805 Universidade de São Paulo, Brasil E-mail: rennan_475@hotmail.com

Arthur Pate de Souza Ferreira ORCID: https://orcid.org/0000-0002-6686-0105 Fundação Oswaldo Cruz, Brasil E-mail: arthurpaterj@gmail.com

Aline Mayara de França Silva ORCID: https://orcid.org/0000-0003-3879-5163 Universidade de Pernambuco, Brasil E-mail: alinem.franca@hotmail.com

Gabriela Madeira Araújo ORCID: https://orcid.org/0000-0003-3999-9547 Universidade de Pernambuco, Brasil E-mail: dra.gabrielamadeira@gmail.com

Neide Pena Coto ORCID: https://orcid.org/0000-0002-3235-0684 Universidade de São Paulo, Brasil E-mail: npcoto@usp.br

Belmiro Cavalcanti do Egito Vasconcelos ORCID: https://orcid.org/0000-0002-6515-1489

Universidade de Pernambuco, Brasil E-mail: belmirovasconcelos@gmail.com

\begin{abstract}
Resumo
Introdução: O uso de equipamentos de proteção individual (EPI) por motociclistas é de grande importância para a prevenção das consequências decorrentes de acidentes. Políticas no Brasil enfatizaram a necessidade do uso de tais equipamentos. O objetivo do estudo foi estimar a frequência e associação do uso de EPI entre os motociclistas e oferecer conselhos sobre o uso adequado. Métodos: Estudo transversal, com uma amostra de 1.516 motociclistas na região metropolitana de Recife, Brasil, entre julho de 2014 e junho de 2015. Foi realizado um questionário sobre gênero, idade, aceitação do teste de alcoolemia, o uso de EPI e cilindrada da motocicleta. Utilizou-se as estatísticas inferenciais ANOVA e teste post-hoc de Tukey. Resultados: $96 \%$ da amostra de motociclistas eram do sexo masculino. Capacetes, sapatos e jaquetas foram os EPI mais utilizados. $92 \%$ da amostra concordou em ouvir conselhos sobre o uso de EPI. Associações significativas foram encontradas entre a idade e os indivíduos que ouviram os conselhos sobre a importância dos EPI, bem como entre o número de EPI utilizados e a cilindrada da motocicleta. Conclusão: Os achados deste estudo enfatizam a importância do uso dos EPI, que são obrigatórios de acordo com a legislação brasileira.
\end{abstract}

Palavras-chave: Ferimentos e Lesões; Acidentes de Trânsito; Equipamento de Proteção Individual; Motocicletas.

\footnotetext{
${ }^{1}$ Autor correspondente: Rennan Luiz Oliveira dos Santos. Av. Prof. Lineu Prestes, 2227 - Cidade Universitária, São Paulo - SP, 05508-000. Departamento de Estomatologia. E-mail: rennan_475@hotmail.com

Conflito de interesses: nada a declarar - Fonte de financiamento: Fundação de Amparo a Ciência e Tecnologia de Pernambuco. Aprovação pela Comissão Nacional de Ética em Pesquisa (CONEP): CAAE 12580413.4.0000.5207.

Contribuição de cada autor: Todos os autores participaram da concepção e delineamento do estudo, da análise estatística, da interpretação dos dados, da redação do artigo, da interpretação dos resultados e da revisão final do artigo.
} 


\begin{abstract}
Introduction: The use of personal protective equipment (PPE) by motorcyclists is of great importance for the prevention of the consequences of accidents. Policies in Brazil emphasized the need to use such equipment. The aim of the study was to estimate the frequency and association of PPE use among motorcyclists and to provide advice on proper use. Methods: A cross-sectional study was conducted with a sample of 1516 motorcyclists in the metropolitan region of Recife, Brazil, between July 2014 and June 2015. A questionnaire was carried out on gender, age, acceptance of alcohol test, use of PPE and motorcycle. The ANOVA inferential statistics and Tukey's post-hoc test were used. Results: $96 \%$ of the sample of motorcyclists were male. Helmets, shoes and jackets were the most used PPE. 92\% of the sample agreed to hear advice about the use of PPE. Significant associations were found between age and individuals who heard the advice about the importance of PPE, as well as between the number of PPE used and the engine capacity of the motorcycle. Conclusion: The findings emphasize the importance of the use of PPE, which are mandatory according to Brazilian legislation.
\end{abstract}

Keywords: Wounds and Injuries; Traffic Accidents; Individual protection equipment; Motorcycles.

\title{
Resumen
}

Introducción: El uso de equipos de protección personal (EPI) por parte de los motociclistas es de gran importancia para la prevención de las consecuencias de los accidentes. Las políticas en Brasil enfatizaron la necesidad de utilizar dicho equipo. El objetivo del estudio fue estimar la frecuencia y asociación del uso de EPP entre motociclistas y brindar asesoramiento sobre su uso adecuado. Métodos: se realizó un estudio transversal con una muestra de 1516 motociclistas de la región metropolitana de Recife, Brasil, entre julio de 2014 y junio de 2015. Se realizó un cuestionario sobre género, edad, aceptación de la prueba de alcohol, uso de EPP y motocicleta. Se utilizó la estadística inferencial ANOVA y la prueba post-hoc de Tukey. Resultados: el $96 \%$ de la muestra de motociclistas fueron hombres. Los cascos, zapatos y chaquetas fueron los EPI más utilizados. El $92 \%$ de la muestra estuvo de acuerdo en escuchar consejos sobre el uso de EPI. Se encontraron asociaciones significativas entre la edad y las personas que escucharon los consejos sobre la importancia de los EPI, así como entre la cantidad de EPI utilizados y la capacidad del motor de la motocicleta. Conclusión: Los hallazgos enfatizan la importancia del uso de EPP, los cuales son obligatorios según la legislación brasileña.

Palabras clave: Heridas y Lesiones; Accidentes de Tránsito; Equipo de protección individual; Motocicletas.

\section{Introdução}

Os traumas causados por acidentes de trânsito vêm aumentando em todo o mundo. Mais de 1,2 milhões de pessoas em todo o mundo morrem vítimas dos acidentes de trânsito a cada ano e mais de $90 \%$ desses óbitos ocorrem em países de baixa e média renda. Além disso, 50 milhões pessoas apresentam sequelas pelos acidentes de trânsito (WHO, 2018). Além do sofrimento das famílias pelas mortes e incapacidades físicas, os custos dos sistemas de saúde com essas vítimas são elevados (Bacchieri, 2011). A Organização Mundial da Saúde (OMS) estima que as perdas anuais devido aos Acidentes de Transporte ultrapassem US\$ 500 bilhões. Um estudo realizado por Barboza Júnior (2021) enfatizou que os homens têm um maior risco de sofrer um acidente com moto e 16,4\% ocasionaram fraturas mais graves nos membros inferiores e superiores (Barboza Júnior, 2021).

A causa das lesões e mortes no trânsito é múltipla e complexa, no entanto, os fatores de risco como dirigir sob a influência de álcool, velocidade excessiva ou inadequada, não utilização de equipamentos de proteção, como o capacete, apresentam alta associação com a ocorrência destes eventos e intervenções focadas nesses fatores podem reduzir a ocorrência e gravidade dos acidentes de trânsito (Oliveira, 2003, Lima, 2004).

Segundo o Departamento Nacional de Trânsito (Denatran), no ano de 2015, o Brasil tem uma frota de 24.301.681 motocicletas, $27,54 \%$ da frota total de veículos.

O uso de motocicletas aumentou 491,1\% no Brasil entre 1998 e 2010, acompanhado de aproximadamente $875 \%$ de aumento de acidentes fatais (Jorge, 1997). Os dados da Associação Nacional de Equipes de Emergência Médica evidenciam que metade de todas as mortes relacionadas à colisão de veículos a motor ocorre logo após o impacto, tornando o socorro médico inviável (Santos, 2008). Assim, ações preventivas podem ajudar a diminuir o número de casos de trauma e morte (Eltorai, 2016). 
O uso de equipamentos de proteção individual (EPI) é um dos principais métodos de prevenção de trauma decorrentes de acidentes de trânsito (Ministry of Labor and Employment, 2011). Por definição, o EPI é qualquer meio ou uso do dispositivo para diminuir riscos que põem em perigo a saúde e segurança de indivíduos durante a execução de uma determinada atividade (Ouellet, 2011). Segundo os dados da Pesquisa Nacional de Saúde realizada em 2013 (IBGE, 2015), os percentuais de indivíduos de 18 anos ou mais que sempre usam o capacete entre condutores e passageiros de motocicletas foram iguais a $83,4 \%$ e $80,1 \%$, respectivamente. Os adultos com nível superior completo apresentaram maiores percentuais de uso de capacete, representando $91,4 \%$ (condutor) 90,1\% (passageiro). Indivíduos da raça-cor branca, das faixas etárias de 40 a 49 e 60 e mais, e Região Sul e Sudeste apresentaram maiores prevalências do uso de capacete (Malta, 2016).

Tal como ocorre no Reino Unido, o Conselho Nacional de Tráfego Nacional (CONTRAN), através do exercício das atribuições concedidas pelo artigo 12 da Lei 9503, estipulou como obrigatório o uso de um capacete tanto por motociclistas como por passageiros para trânsito em estradas públicas (CONTRAN, 2006; Norris, 2013). Além disso, a Lei 11.705 do Código de Tráfego Brasileiro determina a proibição da ingestão de bebidas alcoólicas pelos motoristas. Esta lei é comumente conhecida na "Lei Seca (CONTRAN, 2006).

Como representa um importante problema de saúde pública, o monitoramento da magnitude e tendência da frequência e da gravidade das lesões, das características das vítimas e dos fatores de risco e proteção é fundamental para apoiar políticas públicas para prevenção de lesões e óbitos.

O objetivo do presente estudo é analisar a frequência do uso de equipamentos de proteção individual, segundo características demográficas entre motociclistas na região metropolitana da cidade de Recife, Brasil. Adicionalmente, busca-se realizar uma ação educacional em que se deu orientação sobre a importância de usar os EPI, para uma maior segurança contra traumas e acidentes de trânsito.

\section{Metodologia}

Trata-se de um estudo transversal, utilizando uma amostra não probabilística por conveniência composta por 1.516 motociclistas na região metropolitana da cidade de Recife, Brasil, entre julho de 2014 e junho de 2015. Todas as diretrizes deste estudo foram instruídas pelas autoridades da Operação de Lei Seca para parar a motocicleta do lado da estrada para a verificação da licença e do registro, além de se submeter a um teste de alcoolemia. Esta operação realiza tais ações em diferentes locais da região metropolitana do Recife. Todos os indivíduos da pesquisa assinaram um Termo de Consentimento Livre e Esclarecido (TCLE). Este estudo recebeu aprovação do Comitê de Ética em Pesquisa Humana no processo número 12580413400005207.

Todos os pesquisadores foram submetidos a treinamento antes da fase de campo, administraram um questionário abordando sexo, idade, resultado do teste do bafômetro, o uso de EPI, cilindrada do motociclo e interesse em receber conselhos sobre o uso correto de EPI. Os esclarecimentos foram realizados oralmente e sob a forma de panfletos aos participantes interessados. Além disso, o número de EPIs presentes também foram analisados no presente estudo.

Após o cálculo do tamanho da amostra, todos os dados foram tabulados e os testes estatísticos foram realizados com o auxílio do programa Bioestat 5.3. As estatísticas descritivas foram realizadas em primeiro momento, seguidas de estatísticas inferenciais (ANOVA e teste post hoc de Tukey). Uma margem de erro de 5\% foi utilizada nas decisões dos testes estatísticos. As variáveis foram agrupadas e apresentadas sob a forma de tabelas. 


\section{Resultados}

Dos 1.516 motociclistas entrevistados, aproximadamente $96 \%$ era do sexo masculino e somente $4 \%$ do sexo feminino. $97 \%$ tiveram um resultado negativo no teste de bafômetro, $1,31 \%$ apresentaram resultado positivo no teste de alcoolemia e $1,58 \%$ recusou-se a fazer o teste. Aproximadamente $62 \%$ dos motociclistas tinham idade entre 18 e 29 anos (Tabela 1).

A grande maioria dos motociclistas usavam EPI e apenas um motociclista não estava usando nenhum equipamento. Capacetes, sapatos e casacos foram os EPI mais utilizados, correspondendo a 99,9\%, 60,1\% e $23 \%$ da amostra, respectivamente. Estes mesmos itens foram também o EPI mais utilizado pelos 669 passageiros, com frequências de $89,3 \%$, $58,8 \%$ e $26,7 \%$, respectivamente (Tabela 1 ).

Tabela 1: Perfil de amostra de acordo com o gênero, aceitação do teste de alcoolemia, uso de equipamentos de proteção pessoal e idade.

\begin{tabular}{|c|c|c|c|}
\hline & & $\mathrm{n}$ & $\%$ \\
\hline \multirow[t]{2}{*}{ Sexo } & Masculino & 1457 & 96,1 \\
\hline & Feminino & 59 & 3,9 \\
\hline \multirow[t]{3}{*}{ Teste do Bafômetro } & Positivo & 20 & 1,4 \\
\hline & Recusa & 24 & 1,6 \\
\hline & Negativo & 1472 & 97,0 \\
\hline \multirow[t]{3}{*}{ Idade (em anos) } & $18-29$ & 939 & 61,9 \\
\hline & $30-44$ & 351 & 23,1 \\
\hline & $45-60$ & 226 & 15,0 \\
\hline \multirow[t]{2}{*}{ Uso de pelo menos um EPI } & Sim & 1515 & 99,9 \\
\hline & Não & 1 & 0,1 \\
\hline \multicolumn{4}{|c|}{ Uso de EPI em Piloto (total=1516) } \\
\hline \multirow[t]{2}{*}{ Capacete } & Sim & 1515 & 99,9 \\
\hline & Não & 1 & 0,1 \\
\hline \multirow[t]{2}{*}{ Sapato } & Sim & 911 & 60,1 \\
\hline & Não & 605 & 59,9 \\
\hline \multirow[t]{2}{*}{ Casaco } & Sim & 349 & 23,0 \\
\hline & Não & 1167 & 77,0 \\
\hline \multicolumn{4}{|c|}{ Uso de EPI em passageiro (total=669) } \\
\hline \multirow[t]{2}{*}{ Capacete } & Sim & 597 & 89,3 \\
\hline & Não & 72 & 10,7 \\
\hline \multirow[t]{2}{*}{ Sapato } & Sim & 393 & 58,8 \\
\hline & Não & 276 & 41,2 \\
\hline \multirow[t]{2}{*}{ Casaco } & Sim & 179 & 26,7 \\
\hline & Não & 490 & 73,3 \\
\hline
\end{tabular}

Fonte: Autores.

Dentre os motociclistas abordados na pesquisa, 92\% ouviram os conselhos oferecidos pelos pesquisadores sobre a importância da utilização do EPI. Quase metade das motocicletas tem 125 cilindradas, aproximadamente $36 \%$ tem 150 cilindradas e $15 \%$ tem 250 cilindradas (Tabela 2). 
Research, Society and Development, v. 10, n. 10, e278101018882, 2021

(CC BY 4.0) | ISSN 2525-3409 | DOI: http://dx.doi.org/10.33448/rsd-v10i10.18882

Tabela 2: Perfil da amostra de acordo com a audição de conselhos e capacidade cúbica de motocicleta.

\begin{tabular}{lcc}
\hline & $\mathrm{n}$ & $\%$ \\
\hline Ouviram o conselho & 1396 & 92,0 \\
Sim & 120 & 8,0 \\
Não & & \\
\hline Cilindrada da Motocicleta (cc) & 738 & 48,7 \\
$125 \mathrm{cc}$ & 543 & 35,8 \\
$150 \mathrm{cc}$ & 235 & 15,5 \\
$250 \mathrm{cc}$ & & \\
\hline
\end{tabular}

Fonte: Autores.

Observou-se um gradiente entre a idade e o uso de EPI, ou seja, conforme aumenta a faixa de idade, maior é frequência de utilização de EPI entre os motociclistas. Considerando os grupos etários separadamente, um número médio significativamente maior de EPI foi usado na faixa etária de 30 a 44 anos em comparação com os grupos de 18 a 29 anos e de 45 a 60 anos. Além disso, observou-se uma tendência crescente do número de peças de EPI com um aumento da cilindrada em todas as faixas etárias, não sendo estatisticamente significativo quando analisada a faixa etária de 18 a 29 anos separadamente (Tabelas 3 e 4$)$.

Tabela 3: Relação entre o número de peças de PPE usadas e a idade dos motociclistas.

\begin{tabular}{|c|c|c|c|c|c|c|}
\hline \multirow{3}{*}{$\begin{array}{l}\text { Número de EPI } \\
\text { usados }\end{array}$} & \multicolumn{6}{|c|}{ Faixa de idade (em anos) } \\
\hline & \multicolumn{2}{|c|}{$18-29$} & \multicolumn{2}{|c|}{$30-44$} & \multicolumn{2}{|c|}{$45-60$} \\
\hline & $\mathrm{n}$ & $\%$ & $\mathrm{n}$ & $\%$ & $\mathrm{n}$ & $\%$ \\
\hline 0 & 1 & 0,1 & - & - & - & - \\
\hline 1 & 603 & 64,2 & 18 & 5,1 & - & - \\
\hline 2 & 318 & 33,9 & 105 & 29,9 & 103 & 45,6 \\
\hline 3 & 17 & 1,8 & 105 & 29,9 & 89 & 39,3 \\
\hline 4 & - & - & 106 & 30,2 & 34 & 15,1 \\
\hline 5 & - & - & 17 & 4,9 & - & - \\
\hline Total & 939 & 100 & 351 & 100 & 226 & 100 \\
\hline
\end{tabular}

Fonte: Autores.

Tabela 4: Média e desvio padrão da associação entre o número de peças de EPI usadas e a idade dos motociclistas.

Número de EPI usados

Idade

Média Desvio Padrão p-valor

\begin{tabular}{|c|c|c|c|}
\hline $18-29$ & $1.37 *$ & 0.52 & \\
\hline $30-44$ & $3.00 * *$ & 1.00 & $<0.05$ \\
\hline $45-60$ & $2.70 * * *$ & 0.72 & \\
\hline
\end{tabular}

$*, * *, * * *$ Diferença significativa entre todos os grupos. Fonte: Autores. 


\section{Discussão}

No presente estudo, a grande maioria (aproximadamente 96\%) da amostra era do sexo masculino. Segundo os dados da Pesquisa Nacional de Saúde (PNS) realizada em 2013, a maior parte dos que dirigem motocicleta também é do sexo masculino (74,4\%) (BRASIL, 2013) e do estudo de Corgozinho et al no Distrito federal, que mostrou uma proporção de 96\% de homens motociclistas (Corgozinho, 2017).

De acordo com Veiga, 2014, a legislação brasileira proíbe a ingestão de álcool pelos motoristas, com um nível de álcool superior a $0,1 \mathrm{mg}$ por litro de ar expirado no teste (Veiga, 2004). Apenas 1,4\% dos motociclistas abordados durante a pesquisa tiveram o resultado positivo de alcoolemia, corroborando com um estudo realizado na região metropolitana do Rio de Janeiro, que mostrou que 1,2\% também tiveram resultados positivos. Dentre o total de motociclistas da amostra, $1,6 \%$ se recusaram a fazer o teste, mostrando resultados aproximados a um estudo realizado no Rio de Janeiro em 2010 , que teve $1,1 \% \%$ de recusa (Jomar, 2013) e discordante com resultados de um estudo, na mesma cidade, que teve uma proporção de $4,8 \%$ de recusa (Jomar, 2004).

Os resultados deste estudo evidenciam a alta frequência do uso de EPI. Capacetes, sapatos e casacos foram os EPI mais utilizados, correspondendo a 99,9\%,60,1\% e 23\% da amostra, respectivamente. Estes mesmos itens foram também o EPI mais utilizado pelos 669 passageiros, com frequências de 89,3\%, 58,8\% e 26,7\%, respectivamente. Estes achados vão em concordância com uma PNS (2013), que mostra que os percentuais de uso do capacete entre condutores e passageiros de motocicletas foram iguais a $83,4 \%$ e $80,1 \%$, respectivamente (Malta, 2016).

Em relação ao uso de EPI por faixa de idade, observa-se que neste estudo, conforme aumenta a idade, maior é a proporção de indivíduos que utilizam mais de um EPI, indo em concordância com os dados da PNS (2013), que mostram que $80,7 \%$ dos indivíduos entre 18 e 29 anos sempre usam capacete e esta proporção aumenta para 86,7\% entre os indivíduos de 50 a 59 anos.

92\% dos indivíduos ouviram conselhos sobre a importância dos EPI. Destes, grande parte tiveram uma visão distorcida da importância dos EPI, corroborando com Dos Santos em 2019, afirmando que casacos servem para proteção contra a radiação ultravioleta durante o dia e o frio à noite. Os motociclistas também informaram que o uso de sapatos foi devido às exigências do trabalho, mesmos achados encontrados por Teixeira et al, 2014 investigou as percepções dos entrevistados e encontrou pontos de vista distorcidos quanto ao uso de EPI (Teixeira, 2014). Pordeus et al., 2010 enfatizam o uso de EPI e sua importância para a prevenção de trauma entre motociclistas (Pordeus, 2010).

Os pesquisadores deste projeto ofereceram orientações aos motociclistas e tentaram esclarecer as informações acerca do uso dos EPI. 92\% dos entrevistados receberam informações adequadas sobre os riscos de trauma e a importância do uso de equipamentos de proteção individual. No entanto, são necessárias políticas educacionais mais rígidas e efetivas dirigidas aos motociclistas.

As principais limitações deste artigo estão relacionadas ao desenho da amostra, por ser uma amostragem não probabilística por conveniência. Neste estudo não há como saber se todas as pessoas incluídas na amostra de motociclistas são representativas da população. Outro fator limitante do estudo é um possível viés de informação: os indivíduos responderam o questionário durante uma abordagem da Operação Lei Seca, podendo haver uma inibição do entrevistado.

\section{Considerações Finais}

Este estudo identificou os fatores de risco para traumas e acidentes de trânsito e a importância do uso do EPI por motociclistas. $96 \%$ da amostra de motociclistas eram do sexo masculino. Capacetes, sapatos e jaquetas eram os EPI mais utilizados pelos motociclistas e passageiros. Associações significativas foram encontradas entre a idade e os indivíduos que 
ouviram os conselhos sobre a importância dos EPI, bem como entre o número de EPI utilizados e a cilindrada da motocicleta. Aproximadamente $92 \%$ da amostra concordaram em ouvir conselhos sobre o uso de EPI. Os motociclistas demonstraram pouco conhecimento sobre a importância do equipamento de proteção individual, enfatizando a importância de políticas públicas educacionais efetivas para a prevenção de acidentes no trânsito. Com os resultados encontrados no presente estudo, faz-se necessário a implementação de políticas públicas que priorizem as prevenções de acidentes de trânsito, com a sensibilização da população para o uso dos EPI. Além disso, enfatiza-se a necessidade de estudar mais os fatores de risco para traumas e acidentes de trânsito e a importância do IPI por motociclistas, bem como as associações entre o uso inadequado desses equipamentos e o risco de acidentes mais graves.

\section{Referências}

World Health Organization - Global status report on road safety (2018): WHO: Genebra, 2018. <https://www.who.int/publications-detail/global-statusreport-on-road-safety-2018.>.

Barboza-Junior RS, Golias ARC. (2021). Fraturas provocadas por acidentes de motocicleta. UNINGÁ Journal: 58, e UJ3756. doi: 10.46311/23180579.58.eUJ3756

Bacchieri G, Barros AJD. (2011). Acidentes de trânsito no Brasil de 1998 a 2010: muitas mudanças e poucos resultados. Rev Saude Publica, 45(5):949-963. doi: 10.1590/S0034-89102011005000069.

Oliveira NLB, Souza RMC (2003). Injury diagnosis quality of life among motorcyclists. Traffic Accident Victims Magazine Latino-American Of Nurse by Ribeirão Preto, 11(6): 749-56. doi: 10.1590/S0104-11692003000600008.

Lima DD, Branquinho AL, Magalhães VS, Teixeira BP, Braga BB (2004). Motorcyclists of Brasilia and Goiania, Unpreparedness and vulnerability in traffic. Rev Assoc Bras Med Tráfego, 44:28-31.

Jorge MHPM (1997). À guisa de conclusão. Rev Public Health, 31(4Suppl):51-4. doi: 10.1590/S0034-89101997000500006.

Santos AMR, Moura MEB, Nunes BMVT, Leal CFS, Teles JBM (2008). Profile of victims of trauma motorcycle accident at an emergency public service. Book public health, 24:8. doi: 10.1590/S0102-311X2008000800021.

Eltorai AEM, Simon C, Choi A, Hsia K, Born BT, Daniels DA (2016). Federally mandating motorcycle helmets in the United States. BMC Public Health,16:242. doi: 10.1186/s12889-016-2914-3.

Ministry of Labor and Employment (2011). EPI - Equipamento de Proteção Individual (Personal Protection Equipment) - NR, 6:1-5.

Ouellet JV (2011). Helmet Use and Risk Compensation in Motorcycle Accidents. Traffic Injury prevention, 12:(1) 71-81. doi: $10.1080 / 15389588.2010 .529974$.

Instituto Brasileiro de Geografia e Estatística. Pesquisa Nacional de Saúde 2013. Acesso e Utilização dos Serviços de Saúde, Acidentes e Violências. Rio de Janeiro: Instituto Brasileiro de Geografia e Estatística; 2015. https://biblioteca.ibge.gov.br/visualizacao/livros/liv94074.pdf.

Malta, Deborah Carvalho et al (2016). Lesões no trânsito e uso de equipamento de proteção na população brasileira, segundo estudo de base populacional. Ciênc. saúde coletiva. 21 (2) 399-410. doi: 10.1590/1413-81232015212.23742015.

CONTRAN - Brazilian Council of Transit.Resolution 203 (2006). http://www.denatran.gov.br/download/resolucoes/resolucao203_06.pdf, Accessed January $18,2016$.

Norris E, Myres L (2013). Determinants of Personal Protective Equipment (PPE) use in UK motorcyclists: Exploratory research applying an extended theory of planned behavior (2013). Accident Analysis \& Prevention, 60:219-30. doi: 10.1016/j.aap.2013.09.002.

Corgozinho, Marcelo Moreira; Montagner, Miguel Ângelo (2017). Vulnerabilidade humana no contexto do trânsito motociclístico. Saude soc., São Paulo, 26 (2): 545-555.

Jomar RT, Abreu AMM, Laus MM (2013). Teste do etilômetro e opinião sobre a Operação Lei Seca em amostra de motoristas abordados na cidade do Rio de Janeiro -RJ, 2010 - estudo transversal. Epidemiol Serv Saude. 22(1):121-128. doi: 10.5123/S1679-49742013000100013.

Jomar, Rafael Tavares; Ramos, Dandara de Oliveira; Abreu, Ângela Maria Mendes (2016). Teste do bafômetro: resultados e recusas de motoristas interceptados pela Operação Lei Seca no Rio de Janeiro. Ciênc. saúde coletiva. 21 (12). doi: 10.5123/S1679-49742013000100013.

Viega, FDO et al. (2004). Lei seca: The obstacles in achieving evidence of ways in drunkenness offenses at the wheel. Available at: http://www.sinprfro.com.br./pdf

Dos Santos, RLO et al. (2019). Prevalence of the Use of Individual Protection Equipment and Accidents in Bicycle Users in São Paulo. Revista Ciencias de la Salud. (17): 9 .

Teixeira JRB et al. (2014). Use of personal protective equipment for motorcycle taxi drivers: perception of risks and associated factors. Cad.Saúde Pública, 30(4):885-9. doi: 10.1590/0102-311X00129913.

Pordeus, AMJ et al (2010). Factors associated with the occurrence of motorcycle accidents in the perception of hospitalized motorcycles. Brazilian Journal of Health Promotion, (3): 1-7. doi: 10.5020/2017. 